\title{
LABRANZA MECANIZADA DE PASTURAS MEDIANTE TRES IMPLEMENTOS EN UN ULTISOL Y SUS IMPLICACIONES FÍSICAS E HIDROPEDOLÓGICAS
}

\author{
Manuel E. Camacho ${ }^{1 / *}$, Rafael Mata*, Warren Forsythe*, \\ Palabras clave: Compactación de suelo; infiltración; mecanización; pasturas; Ultisoles; Hidropedología. \\ Keywords: Soil compaction; infiltration; grasslands; Ultisols; Hydropedology.
}

Recibido: 06/02/15

Aceptado: 16/06/15

\section{RESUMEN}

Mediante un estudio en el campo, se determinó el efecto de la labranza mecanizada con 3 implementos sobre la compactación, las variables físicas e hidropedológicas de un Ultisol dedicado a la ganadería, ubicado en San Mateo, Alajuela. Se seleccionó un lote de aproximadamente 10 $000 \mathrm{~m}^{2}$, se dividió en 16 parcelas iguales $(650$ $\mathrm{m}^{2}$ cada una), y se aleatorizó la mecanización con 3 diferentes implementos correspondientes a los tratamientos, a partir de una parcela y un diseño experimental irrestricto al azar. Como tratamientos se estableció un área sin labranza (T), y labranza mediante un palín mecánico (PM), un arado de cincel (AC) o un subsolador (S). Cuarenta días después de la aplicación de los tratamientos se determinó la resistencia a la penetración cada $5 \mathrm{~cm}$ hasta los $50 \mathrm{~cm}$ de profundidad, y la humedad gravimétrica, densidad aparente y de partículas, infiltración y conductividad hidráulica fueron evaluadas, todas ellas en los primeros $10 \mathrm{~cm}$ de profundidad. Se encontró un descenso en la compactación del suelo, expresada como resistencia a la penetración, para los tratamientos de mecanización, y reportó el tratamiento palín mecánico (PM) con los valores más bajos. Este mismo tratamiento incrementó la infiltración acumulada $(38,70 \pm 3,60 \mathrm{~mm}$ a los $150 \mathrm{~min}) \mathrm{de}$ manera significativa, al comparar con la obtenida

1 Autor para correspondencia. Correo electrónico: manuel.camacho87@gmail.com

\section{ABSTRACT}

Grassland soil tillage by three implements in an Ultisol and its physical and hydropedological implications. A field study was conducted to test the effects of soil tillage with 3 different implements on compaction, physical and hydropedological properties of an Ultisol under cattle production, located in San Mateo, Alajuela. An area of approximately 10000 $\mathrm{m}^{2}$ was selected and divided into 16 plots $\left(650 \mathrm{~m}^{2}\right.$ each) and was tilled with 3 different implements corresponding to the treatments, following an unrestricted random experimental design, with a plot as experimental unit. Soil without tillage (T), tillage by spader plow (PM), tillage by chisel plow (C) or tillage by subsoiler (S) were established as treatments. Forty days after tillage treatments, soil penetration resistance every $5 \mathrm{~cm}$ up to $50 \mathrm{~cm}$ deep was assessed, and gravimetric moisture content, bulk and particle density, water infiltration and hydraulic conductivity, all of them up to the first $10 \mathrm{~cm}$ deep, all of them were measured. Soil compaction, expressed as soil penetration resistance, was reduced by tillage treatments; the lowest values for soil compaction were found in the spader plow treatment (PM). This same treatment enhanced cumulated infiltration $(38.70 \pm 3.60 \mathrm{~mm}$ at $150 \mathrm{~min})$ significantly, comparing with those obtained in $\mathrm{T}$ treatment

Centro de investigaciones Agronómicas. Universidad de Costa Rica. San José. Costa Rica. 
en el tratamiento testigo $(0,09 \pm 0,02 \mathrm{~mm}$ a los 150 min). No se encontró diferencias significativas entre los tratamientos de mecanización para densidad aparente, porosidad y espacio aéreo, pero sí con respecto al tratamiento T. El tratamiento subsolador (S) propició los valores más elevados de conductividad hidráulica, sin diferencias estadísticas del resto de tratamientos ( $p>0,05)$.

\section{INTRODUCCIÓN}

Los sistemas pastoriles representan una muy importante actividad económica a nivel nacional. Sin embargo en los últimos años se ha reportado una reducción significativa de las áreas dedicadas a la ganadería. Para 1988 se habían censado 2420118 ha, y 1349628 ha en el 2000, con un incremento de carga animal de 0,70 a 0,77 unidades animales ha ${ }^{-1}$ para los mismos años respectivamente (MAG 2000). Dicho aspecto sugiere un uso más intensivo de las tierras dedicadas a la ganadería.

Ya para el 2012, se reportó un total de 1 265 107, 7 Ha dedicadas al pastoreo, y la carga animal total incrementó a 0,99 unidades animales.ha ${ }^{-1}$ (CORFOGA 2013).

Aunado a lo anterior y la presión de producción de ganado bovino, resulta en el sobre pastoreo que conlleva a la degradación progresiva de los suelos sobre los cuales se desarrollan dichas actividades (Forsythe 1997, Wheeler et ál. 2002), además trasciende en una reducción de la cobertura y en los rendimientos esperados (Kauffman y Krueger 1984). Los efectos del ganado bovino sobre el suelo han sido estudiados por múltiples autores (Kauffman y Krueger 1984, Warren et ál. 1986) y es definido como un agente modificador del suelo y el paisaje (Trimble y Mendel 1995).

Un efecto directo de estos sistemas de producción es la compactación, la cual se define
$(0.09 \pm 0.02 \mathrm{~mm}$ at $150 \mathrm{~min})$. No significant differences were found among tillage treatments for bulk density, total porosity and airspace, but comparing with control treatment $(\mathrm{T})$ they were found. Subsoiler treatment (S) favored the highest values for hydraulic conductivity, but no significant differences with the other treatments were found $(p>0.05)$.

como un incremento en la densidad del suelo producto de presiones o cargas ejercidas sobre el mismo (Baver et ál. 1972) las cuales provocan la compresión de partículas solidas, gases y líquidos dentro del espacio poroso, que a su vez generan un reacomodo estructural del suelo (Alvarado y Agüero 1983). Este fenómeno ha sido reportado por otros autores como ente degradador de la calidad del suelo (Agüero y Alvarado 1983, Forsythe 1997, Dec et ál. 2011, Dec et ál. 2012).

Forsythe et ál. (2005) mencionan varios parámetros físicos por los cuales es posible evaluar la compactación de un suelo, entre ellos la porosidad, la densidad aparente, la infiltración, y la resistencia a la penetración, donde este último presenta el mejor parámetro para determinar el grado de compactación. Estos mismos han sido reportados por otros autores (Kauffman y Krueger 1984, Warren et ál. 1986, Sharrow 2007, Dec et ál. 2012) como índices de compactación y adensamiento del suelo. Parte del problema de compactación en pasturas radica en la disminución de la profundidad de raíces por parte de las plantas, obteniéndose como resultado un descenso en el desarrollo de biomasa radical y por ende en la biomasa aérea de la planta (Paripovic 2011). Bajo estas condiciones en dichos sistemas, se han reportado descensos en la profundidad de las raíces directamente relacionados al incremento de la densidad aparente del suelo (Skinner et ál. 
2009), lo que genera disminuciones de hasta un $75 \%$ de dicha profundidad en comparación con las desarrolladas en suelos menos compactados.

Desde el punto de vista pedológico e hidrológico, la compactación del suelo se ve fuertemente involucrada en los múltiples procesos de la génesis y la evolución de un suelo, así como en los fenómenos del movimiento y retención del agua en el mismo (Letey 1985, Da Silva et ál. 1994). Dentro de los anteriores destacan la capacidad de drenaje y movimiento del agua, la aireación y reacciones oxido-reducción en el perfil del suelo, así como el adensamiento y formación de horizontes endurecidos, responsables de elementos formativos dentro del nombre taxonómico de un suelo (Buol et ál. 2011). Por lo anterior, conviene estudiar la compactación del suelo de una forma integrada e interdisciplinaria, a partir de conceptos tanto pedológicos como hidrológicos.

Recientemente se ha venido desarrollado la hidropedología, una ciencia interdisciplinaria que describe la interacción entre los procesos pedológicos e hidrológicos (Lin 2003, Lin et ál. 2006, Pachepsky et ál. 2006). Esta disciplina se fundamenta en 2 aspectos generales: 1) el rol del paisaje y la arquitectura del suelo en los procesos hidrológicos, y 2) el efecto de los procesos hidrológicos sobre la génesis y evolución del suelo, variabilidad y funcionalidad (Lin 2012). Bouma (2006) describe procesos relacionados con la hidropedología e importancia en la investigación y políticas ambientales. Destaca entre ellos el flujo de derivación, la conductividad hidráulica saturada, los niveles de tabla de agua, la accesibilidad de agua, e hidrofobicidad del suelo. La conductividad hidráulica es una propiedad de suma importancia en la hidropedología, la cual es descrita desde este enfoque como el comportamiento hidráulico del suelo en función de características propias debidas a su proceso de génesis como el desarrollo de la estructura o la micro morfología del perfil (Bouma 2006). Por ello, variaciones en esta propiedad producto de manejo del suelo o prácticas asociadas a cultivos podrían tener repercusiones no solo sobre el comportamiento mecánico del suelo, sino también sobre los procesos de su desarrollo y evolución.

En Costa Rica, los trabajos sobre compactación de suelos y sus repercusiones sobre el desarrollo y rendimiento de los cultivos son escasos (Alvarado y Agüero 1983, Forsythe y Tafur 1985, Forsythe y Schweizer 2001, Forsythe et ál. 2005). Sumado a lo anterior, no se han encontrado estudios sobre la relación de parámetros físicos del suelo como la compactación y la infiltración, y menos aún, sobre el estudio de las propiedades hidropedológicas.

Basado en lo anterior, el objetivo del presente trabajo fue determinar el efecto de 3 implementos de labranza sobre la compactación y algunas propiedades físicas e hidropedológicas de un Ultisol dedicado a la ganadería en San Mateo de Alajuela, Costa Rica.

\section{MATERIALES Y MÉTODOS}

El experimento se llevó a cabo en San Mateo, Alajuela (Figura 1). Se localizó un terreno dedicado a la ganadería con un área de aproximadamente de una hectárea $\left(10000 \mathrm{~m}^{2}\right)$. El terreno se encontró ubicado en el pie de monte de los cerros del Aguacate (Bergoeing 1998), con una gradiente de 0,03 . Se clasifica dentro del sistema de vida de Holdridge (1967) bosque húmedo tropical (Bh-T), y el drenaje del sitio conduce a un afluente del río Jesús María. El sitio de estudio se encuentra ubicado en la zona climática Pacífico Norte, con precipitaciones que corresponden al denominado régimen de precipitación del Pacífico, con 2 periodos definidos, la estación lluviosa y la estación seca (Solano y Villalobos 2012). Los parámetros climáticos se describen en la Figura 2.

El suelo del sitio se clasificó taxonómicamente como un Andic Haplohumults según el sistema USDA (Soil Survey Staff 2014), las características del suelo se presentan en el Cuadro 1. El terreno se encontraba sembrado con pasto Bracharia brizantha, especie con crecimiento cespitoso y reproducción por estolones, la cual presenta de manera general un $80 \%$ de la biomasa de raíces distribuido 

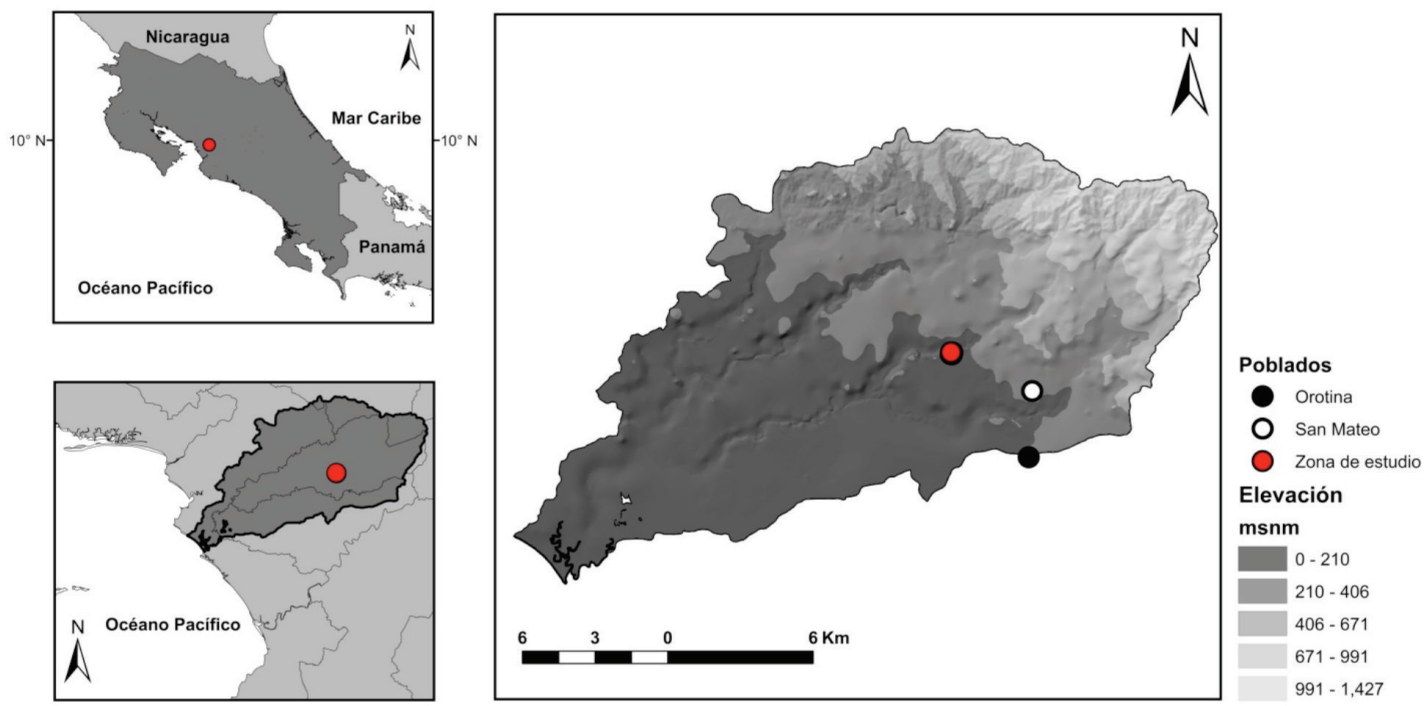

Fig. 1. Localización geográfica del sitio de estudio y muestreo en la cuenca del río Jesús María, San Mateo. Alajuela. 2015.

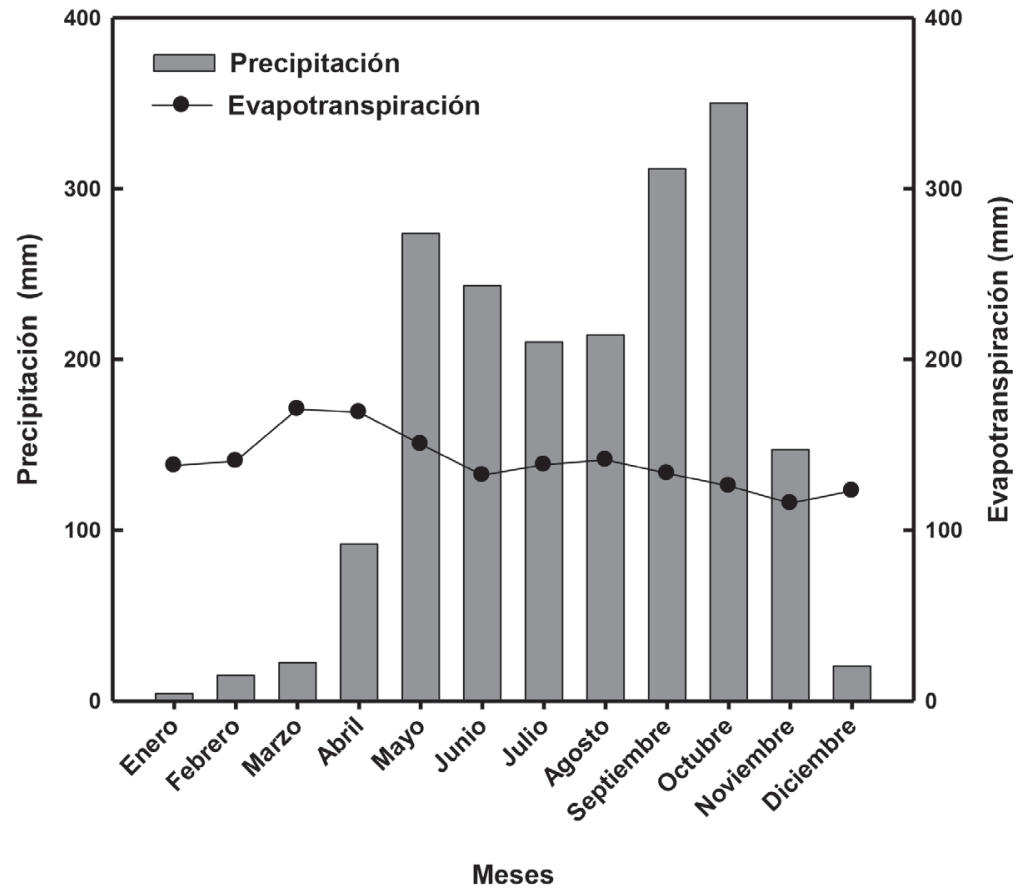

Fig. 2. Precipitación media mensual y evapotranspiración potencial estimada para la región de la cuenca del río Jesús María. Alajuela. 2015. Evapotranspiración calculada según Hargreaves y Samani (1985). Datos climáticos obtenidos de una estación meteorológica ubicada en Orotina ( $09^{\circ} 56$ N; 8422 O) en un periodo de 18 años (1995-2013). 


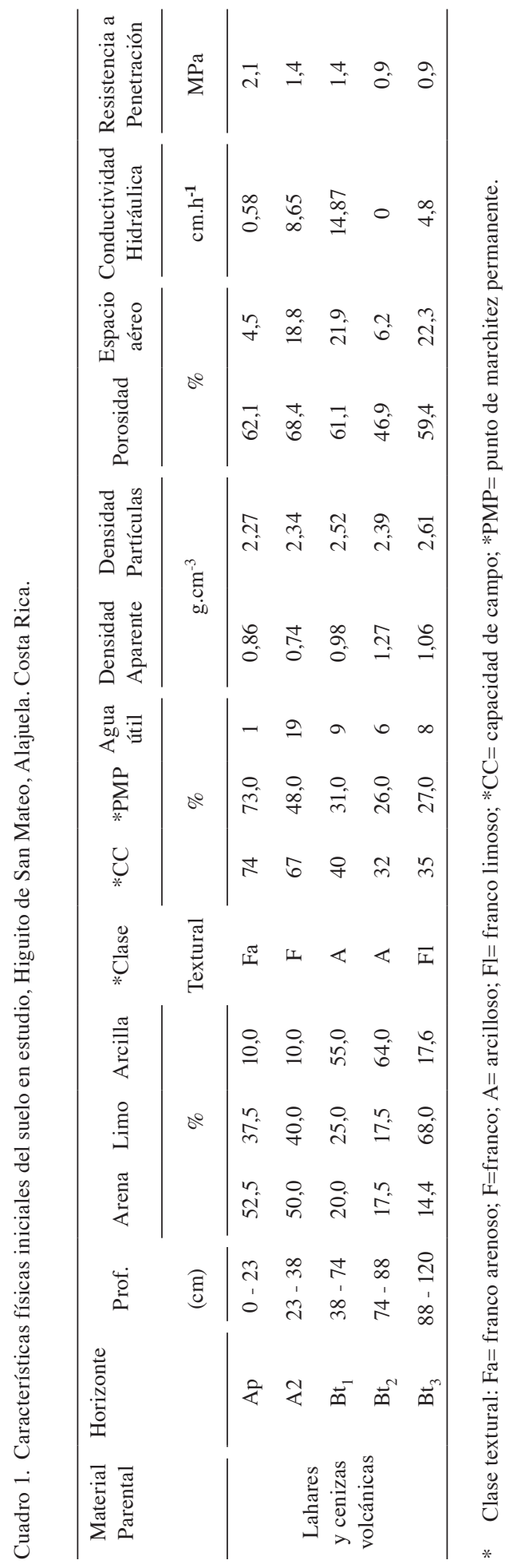

Agronomía Costarricense 39(3): 101-115. ISSN:0377-9424 / 2015 
en los primeros $30 \mathrm{~cm}$ del perfil del suelo y baja tolerancia a la sequía (Guenni et ál. 2002). Dicho terreno se dividió en 16 parcelas iguales correspondientes a 4 repeticiones para 4 tratamientos.

Dichos tratamientos consistieron en la mecanización del suelo mediante 3 implementos de labranza: el palín mecánico o azada mecánica (PM), el arado de cincel rígido (AC) y el subsolador (S), los cuales fueron acoplados a un tractor Fiat ${ }^{\circledR} 100-90(90 \mathrm{cv})$. Las características técnicas de los 3 implementos se describen en detalle en Alvarado (2004). Como tratamiento testigo se establecieron sitios sin mecanizar. Cuarenta días posteriores se realizaron las evaluaciones correspondientes a los parámetros físicos e hidropedológicos, bajo condiciones de humedad a capacidad de campo.

\section{Diseño experimental}

El experimento se estableció a nivel de campo, en un diseño experimental irrestricto al azar, con 4 tratamientos y 4 repeticiones por cada tratamiento. Se estableció como unidad experimental una parcela de $650 \mathrm{~m}^{2}$.

Los tratamientos que se aplicaron fueron los siguientes:

- $\quad$ Testigo sin labranza (T).

- Labranza mediante palín mecánico a 35 $\mathrm{cm}$ de profundidad (PM), a una velocidad de trabajo de $0,6 \mathrm{~km} \cdot \mathrm{h}^{-1}$ y $540 \mathrm{rpm}$ en la toma de fuerza.

- Labranza mediante arado de cincel a 30 $\mathrm{cm}$ de profundidad (AC), a una velocidad de trabajo de $7 \mathrm{~km} . \mathrm{h}^{-1}$ con un distanciamiento entre cinceles de $25 \mathrm{~cm}$.

- Labranza mediante subsolador a $40 \mathrm{~cm}$ de profundidad (S), a una velocidad de trabajo de $7 \mathrm{~km} \cdot \mathrm{h}^{-1}$, con un distanciamiento entre subsoladores de $50 \mathrm{~cm}$.

\section{Variables de compactación, físicas e hidropedológicas}

Para cada parcela se determinó la resistencia a la penetración cada $5 \mathrm{~cm}$ hasta alcanzar
$50 \mathrm{~cm}$ de profundidad como variable directa de compactación mediante un penetrómetro Eijkelkamp ${ }^{\circledR}$ empleado el cono \# 2, el cual tiene un área basal de $2 \mathrm{~cm}^{2}$ y un ángulo apical de $60^{\circ}$. Como variables físicas se determinó la densidad aparente, densidad de partículas del suelo y la humedad gravimétrica mediante la toma de muestras inalteradas en cilindros de 5 x 5 cm según lo sugerido por Forsythe (1985), estas determinaciones se realizaron en los primeros 10 $\mathrm{cm}$ del horizonte superficial (Ap 0-23 $\mathrm{cm}$ de profundidad). A partir de ambos valores de densidad y la humedad se calculó la porosidad total y el espacio aéreo de acuerdo con Forsythe (1985). Como variables hidropedológicas se evaluó la infiltración del suelo mediante la metodología de los anillos concéntricos propuesta por Bower (1986) y la conductividad hidráulica de acuerdo con Forsythe (1985).

Para cada parcela se realizaron 6 mediciones de resistencia a la penetración, también se colectaron 6 muestras indisturbadas para el análisis de densidad aparente densidad de partículas y conductividad hidráulica, y se realizó una determinación de infiltración.

\section{Análisis estadístico}

Mediante el uso del paquete estadístico InfoStat ${ }^{\circledR}$, se realizó un análisis de varianza "ANDEVA" para las variables densidad aparente, porosidad total, espacio aéreo y conductividad hidráulica, se utilizó la prueba de LDS Fisher para separar las medias en las variables que presenten diferencias mínimas significativas con $\mathrm{p}<0,05$. Para la evaluación de la compactación se aplicó un ANDEVA factorial con interacción entre profundidad y los tratamientos, y se utilizó la prueba LDS Fisher para determinar diferencias significativas entre los tratamientos $(\mathrm{p}<0,05)$.

Para el análisis de infiltración se determinó las constantes de la ecuación de Kostiakov (1932) para cada tratamiento mediante un modelo regresión de forma potencial con la velocidad de infiltración $\left(\mathrm{cm} . \mathrm{min}^{-1}\right)$ como variable dependiente y el tiempo ( $\mathrm{min}$ ) como variable independiente: 


$$
\mathrm{Q}=\alpha \tau^{\beta}
$$

Donde $Q$ corresponde a la velocidad de infiltración; $\tau$ el tiempo; $\alpha$ y $\beta$ constantes de regresión.

Para la determinación de la infiltración acumulada $(\mathrm{cm})$, se integró la ecuación anterior en los límites de tiempo $\mathrm{t}=0$ y $\mathrm{t}=1$ para generar la siguiente ecuación:

$$
I=\int_{0}^{\tau} Q d \tau=\int_{0}^{\tau} \alpha \tau^{\beta}=\frac{\alpha \tau^{\beta+1}}{\beta+1}
$$

Donde $I$ es la infiltración acumulada en $\mathrm{cm} ; \alpha$ y $\beta$ son las constantes obtenidas en la regresión, $\tau$ es el tiempo en minutos.

\section{RESULTADOS Y DISCUSIÓN}

\section{Efecto de los tratamientos de mecanización sobre la compactación del suelo}

Los tratamientos de labranza mecanizada redujeron la compactación del suelo, estimada por la resistencia a la penetración. Se observa que en los primeros $5 \mathrm{~cm}$, no existe diferencia significativa ( $L D S=0,22 \mathrm{MPa} ; \mathrm{p}<0,05)$ en los valores de resistencia a la penetración entre implementos pero sí entre el tratamiento testigo y el suelo arado por los implementos (Figura 3).

Conforme aumentó la profundidad del suelo los valores de resistencia a la penetración reportados para cada tratamiento comenzaron a incrementar. El tratamiento PM presenta valores menores a $1 \mathrm{MPa}$ hasta una profundidad de 40 $\mathrm{cm}$, y a partir de $15 \mathrm{~cm}$ este tratamiento presenta valores estadísticamente distintos de los obtenidos por AC y S, para posteriormente obtener valores muy cercanos a $1 \mathrm{MPa}$ a una profundidad de $50 \mathrm{~cm}$, de forma similar a lo obtenido por los otros 2 tratamientos. A esta misma profundidad no se observa una diferencia de los tratamientos de labranza mecanizada comparados con el testigo (LDS=0,22 MPa; $\mathrm{p}<0,05$ ).

El tratamiento $\mathrm{S}$ presentó valores de resistencia a la penetración similar a PM durante los primeros $15 \mathrm{~cm}$, a partir de esta profundidad dichos valores incrementan al punto que no presentan diferencias significativas con los valores del tratamiento AC y con el $\mathrm{T}$ al alcanzar los 30 cm (Figura 3). De manera general se observó el efecto de los distintos implementos de labranza, obteniéndose diferencias significativas entre los valores de resistencia a la penetración encontrados en el tratamiento testigo y los obtenidos en los 3 tratamientos en los primeros $20 \mathrm{~cm}(\mathrm{p}<0,05)$.

Destaca el tratamiento PM, el cual dio los valores más bajos de resistencia a la penetración hasta una profundidad de $40 \mathrm{~cm}$, con diferencias significativas con respecto a los otros 2 tratamientos de mecanización y al tratamiento testigo (Figura 3). Este tratamiento reportó valores de resistencia inferiores a $0,94 \mathrm{MPa}$ dentro $50 \mathrm{~cm}$ de profundidad evaluados, y según Listo (2009) este valor de resistencia corresponde al límite inferior para el ámbito de clasificación de un suelo como moderadamente compactado. Lo anterior podría explicarse según Alvarado (2004), quien expone que el funcionamiento del palín mecánico produce un volteo mínimo del suelo y requiere menos pases del implemento, lo contribuye en la reducción de la compactación y evita la formación del pie de arado. Otros autores reportan estas mismas ventajas del uso de este implemento, además mencionan la conservación de la estructura del suelo producto de la baja disturbación de los agregados (Sarrantonio y Molloy 2003, Pritchett et ál. 2011).

Por otro lado, Licht y Al-Kaisi (2005) evaluaron implementos de labranza como el arado de cincel y arado de franja contra sitios sin mecanizar. Encontraron que a una profundidad de $0-10$ $\mathrm{cm}$ el tratamiento de arado de cincel reportó valores más bajos de resistencia a la penetración que tratamientos sin mecanizar y labranza en franja. Pierce et ál. (1992) estudiaron diferentes sistemas de labranza en un suelo fine-loamy, mixed, mesic Typic Hapludalf; encontraron reducciones significativas en la resistencia a la penetración (0,5 $\mathrm{MPa})$ para los tratamientos de subsolado en comparación con el tratamiento sin mecanización (3 MPa). Martínez y Zinck (2004) reportaron 


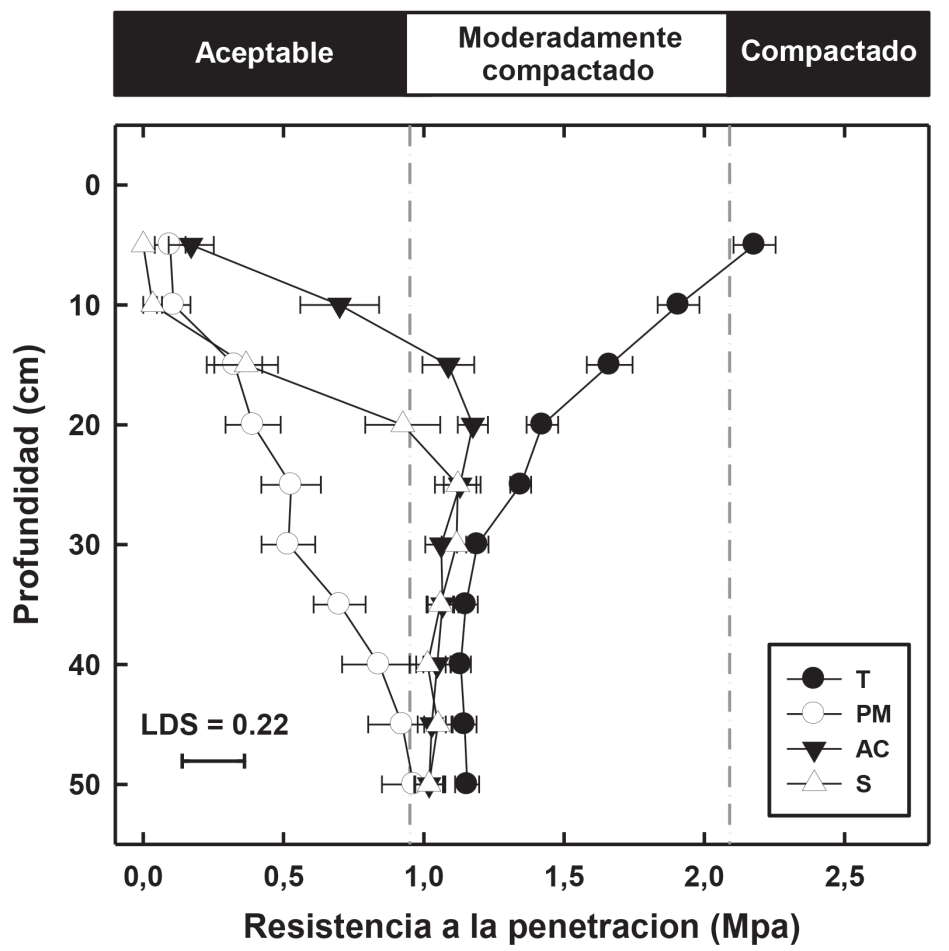

Fig. 3. Efecto de distintos equipos de labranza sobre la resistencia a la penetración de un Andic Haplohumults evaluada hasta $50 \mathrm{~cm}$ de profundidad. San Mateo. Costa Rica. Barras de error estimadas como error estándar. LDS= diferencia mínima significativa estimada para la separación de medias para la interacción profundidad x tratamiento en el análisis estadístico $(\mathrm{p}<0,05)$. Ámbitos de compactación tomados de Listo (2009).

valores de hasta 4,5 MPa en los primeros $20 \mathrm{~cm}$ en pasturas de 9 años. Destacan el incremento en la resistencia a la penetración producto del pisoteo del ganado sobre el suelo tras un periodo largo de pastoreo. Resultados similares fueron reportados por Agüero y Alvarado (1983) para suelos bajo pastoreo en la provincia de Guanacaste, Costa Rica.

\section{Efecto de los tratamientos de mecanización sobre las variables físicas del suelo}

Los tratamientos de labranza mecanizada afectaron de manera significativa las variables físicas del suelo evaluado (Figura 4). Se observó un descenso de la densidad aparente así como un incremento en la porosidad total y el espacio aéreo en los primeros $10 \mathrm{~cm}(\mathrm{p}<0,05)$. Para estas variables no se encontró diferencias significativas entre los implementos pero sí con respecto al testigo (Figura 4).

El resultado más evidente corresponde al incremento en el espacio aéreo obtenido en los 3 implementos, el cual corresponde a un incremento del $50 \%$ con respecto al testigo (Figura 4). Esto se debe probablemente al efecto del implemento en la ruptura de los macro-agregados, el cual los secciona en partes más pequeñas que reducen la densidad aparente del suelo, e incrementan el espacio poroso. Para este parámetro del suelo la diferencia entre los 3 tratamientos de labranza mecanizada es mínima. Sin embargo, el espacio aéreo obtenido para el tratamiento testigo no fue 


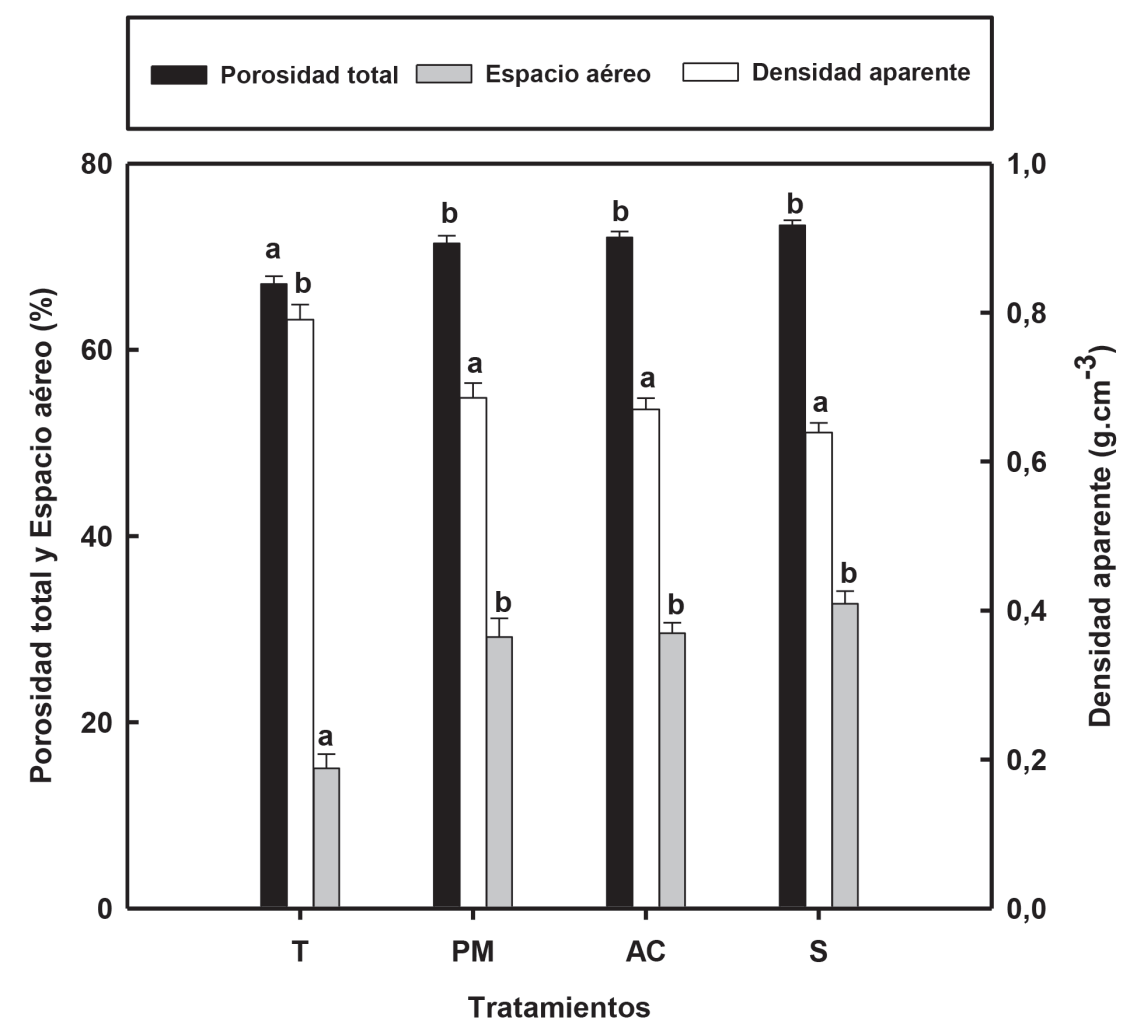

Fig. 4. Efecto de distintos equipos de labranza sobre los distintos parámetros físicos de un Andic Haplohumults en los primeros $10 \mathrm{~cm}$ del horizonte Ap de (0-23 cm de profundidad). San Mateo. Costa Rica. Letras distintas denotan diferencia significativa de acuerdo con prueba LSD Fisher $(\mathrm{p}<0,05)$.

limitante para el desarrollo de las plantas según lo reportado en literatura (Baver et ál. 1972).

Al respecto Álvarez y Steinbach (2009) en un estudio de revisión de resultados de 35 experimentos de mecanización encontraron que los valores de densidad aparente fueron significativamente más bajos en tratamientos de mecanización convencional que los reportados para los sitios bajo cero labranza, evaluados en los primeros $25 \mathrm{~cm}$ de profundidad $(\mathrm{p}<0,05)$. Kay y VandenBygaart (2002) mencionan la reducción de la porosidad en suelos que pasan de un manejo bajo mecanización convencional a uno sin mecanización o cero labranza. Lipiec et ál. (2006) encontraron en un Eutric Fluvisol los valores más elevados de porosidad en un tratamiento de mecanización, en comparación con el tratamiento cero labranza, el cual reportó los más bajos.

\section{Efecto de los tratamientos de mecanización sobre las variables hidropedológicas}

Se obtuvo como resultado que los tratamientos de labranza mecanizada incrementaron la infiltración acumulada determinada posteriormente en las parcelas respectivas. A un tiempo de 150 min de evaluación, la infiltración acumulada estimada para el tratamiento PM $(38,70 \pm 3,60 \mathrm{~mm})$ incrementó de manera significativa con respecto al valor estimado para el tratamiento testigo $(0,09 \pm 0,02 \mathrm{~mm})$ a ese mismo tiempo (Figura 5). Este incremento 


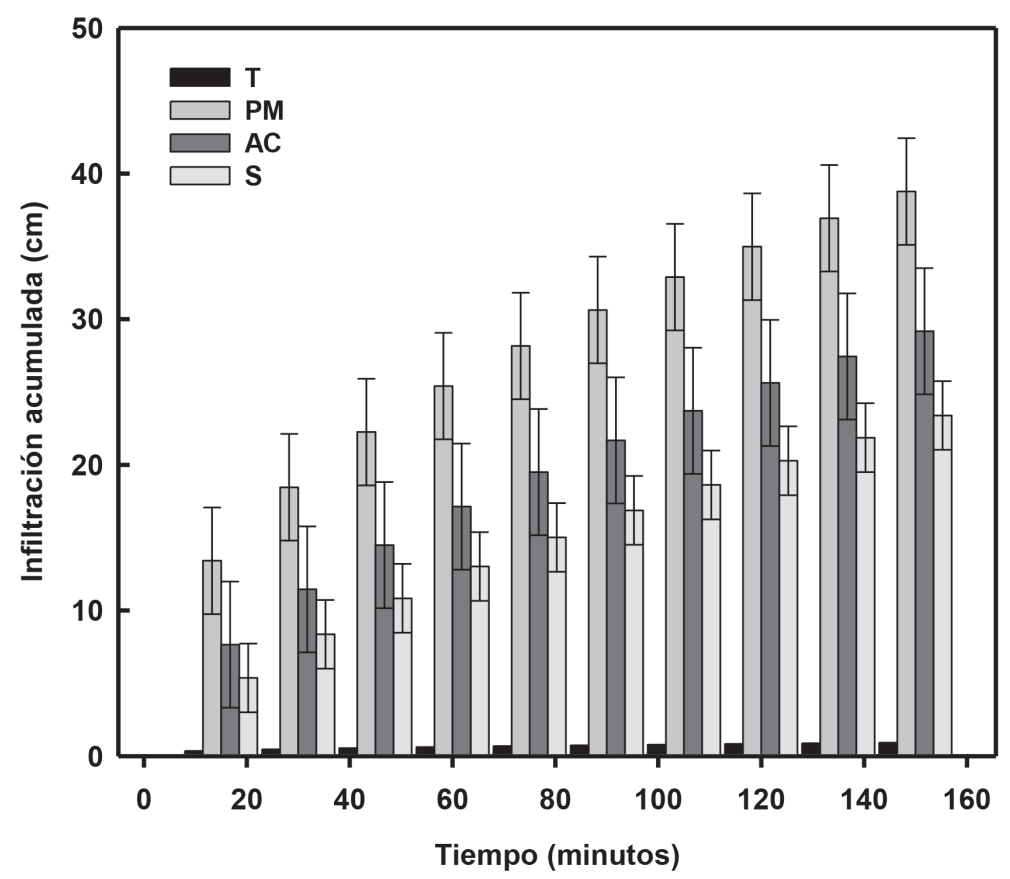

Fig. 5. Efecto de distintos equipos de labranza sobre la infiltración acumulada de un Andic Haplohumults evaluada en los primeros $10 \mathrm{~cm}$ del horizonte Ap de (0-23 cm de profundidad). San Mateo. Costa Rica. Barras de error estimadas como error estándar.

fue de casi 41 veces el valor de infiltración acumulada del testigo a lo largo del tiempo de evaluación hasta alcanzar la infiltración estabilizada. Los otros 2 implementos también incrementaron la infiltración acumulada pero en valores más bajos que los obtenidos por el tratamiento del PM (Figura 5). Esto se debe posiblemente al modo de acción del instrumento, que genera un volteo mínimo y ruptura superficial del suelo en agregados más finos de forma homogénea, que permite mayor separación entre los agregados y la continuidad de los macro poros del suelo que facilita la infiltración del agua (Alvarado 2004).

Al respecto, Alegre et ál. (1991) resumió gran cantidad de resultados de ensayos sobre labranza convencional, labranza de conservación y cero labranza realizados en Latinoamérica y encontró bajas tasas de infiltración en los sitios bajo mínima o cero labranza, en comparación con suelos bajo labranza convencional con subsolado y/o arado de cincel. Resultados similares han sido reportados por otros autores (Horne et ál. 1992, Gómez et ál. 1999, Lipiec et ál. 2006, Strudley et ál. 2008).

Los bajos valores de infiltración acumulada obtenidos para el tratamiento $\mathrm{T}$ se deben probablemente a un uso intensivo del sitio como actividad ganadera. Pietola et ál. (2005) estudiaron el efecto del pisoteo del ganado sobre las propiedades hidráulicas del suelo, que presentó reducciones significativas de la infiltración en sitios con mayor actividad del ganado. Resultados y conclusiones similares se reportan por otros autores (Warren et ál. 1986, Trimble y Mendel 1995, Mwendera y Saleem 1997).

Por otro lado los valores de conductividad hidráulica obtenidos en los primeros $10 \mathrm{~cm}$ del horizonte superficial (Ap) de las parcelas se comportaron de manera distinta a los resultados 
anteriores. Los valores promedio de conductividad hidráulica obtenidos para cada tratamiento no presentaron diferencias estadísticamente significativas $(p>0,05)$ entre ellos (Figura 6). Los



Fig. 6. Efecto de distintos equipos de labranza sobre la conductividad hidráulica de un Andic Haplohumults en los primeros $10 \mathrm{~cm}$ del horizonte Ap de (0- $23 \mathrm{~cm}$ de profundidad). San Mateo. Costa Rica.

* Sin diferencias significativas de acuerdo con prueba LSD Fisher ( $p>0,05)$.

valores mencionados anteriormente presentaron la siguiente tendencia: Tratamiento $\mathrm{S}\left(102,9 \mathrm{~cm} \cdot \mathrm{h}^{-}\right.$ $\left.{ }^{1}\right), \operatorname{PM}\left(79,9 \mathrm{~cm} \cdot \mathrm{h}^{-1}\right), \mathrm{AC}\left(37,8 \mathrm{~cm} \cdot \mathrm{h}^{-1}\right)$ y por último $\mathrm{T}\left(22,4 \mathrm{~cm} \cdot \mathrm{h}^{-1}\right)$. Estos resultados se deben probablemente a la gran variabilidad intrínseca de este parámetro dentro de un mismo suelo, vista como los errores estándar estimados para la media de cada tratamiento (Figura 6). Lo anterior concuerda con lo reportado por otros autores (Mubarak et ál. 2010, Rienzner y Gandolfi 2014).

Esta variabilidad de la conductividad hidráulica en un suelo saturado, está más asociada a la micromorfología de los poros que a la porosidad total del suelo (Nakano y Miyazaki 2005, Dexter y Richard 2009, Ingelmo et ál. 2011), es por esta razón que se pueden encontrar valores de conductividad mayores en un sector de la profundidad del suelo inmediata a la capa superficial (subsuelo) afectada por compactación que los obtenidos en la parte más superficial del suelo donde existe disturbación del sistema de poros por labranza (Dexter et ál. 2004). Sin embargo, existen gran cantidad de estudios que reportan variaciones de la conductividad hidráulica en suelo saturado asociadas a la mecanización. Pierce et ál. (1992) encontraron incrementos en la conductividad hidráulica de flujo saturado en 3 profundidades del suelo en sitios mecanizados con subsolador, con valores estadísticamente superiores al tratamiento sin mecanizar.

Xu y Mermoud (2003) elaboraron ensayos de mecanización convencional y sin labranza y 
un subsolador para modelar la dinámica del agua inferida de la conductividad hidráulica del suelo bajo estos 3 manejos. Los autores encontraron valores superiores de conductividad hidráulica durante los primeros días de evaluación en el tratamiento de subsolador. Otros autores reportan resultados similares (Sojka et ál. 1997, Drewry y Paton 2000).

Los tratamientos de mecanización, tuvieron en general un efecto positivo sobre las variables hidropedológicas del sitio en estudio, ya que incrementan la infiltración acumulada con respecto al tratamiento testigo. Estos efectos podrían tener beneficios a mediano y largo plazo, como el incremento en la infiltración y la capacidad de almacenamiento de agua en el suelo (Guzha 2004), que redujo a la vez, el agua de escorrentía y mitigó el proceso de erosión laminar, muy común en estos sistemas de pasturas intensivos donde los valores de escorrentía son elevados (Trimble y Mendel 1995).

Sumado a lo anterior, el mejoramiento de las propiedades físicas del suelo podría generar beneficios en la actividad ganadera, tales como el incremento el rendimiento del pasto expresado como biomasa seca producto de un incremento en la porosidad y el espacio aéreo, ambos claves en la aireación del suelo y por tanto en el desarrollo radical y de la parte aérea en las gramíneas. Para futuros estudios de labranza mecanizada en pasturas, se recomienda la evaluación de parámetros de crecimiento y rendimiento del pasto, con la finalidad de evaluar el posible efecto de la mejora del suelo sobre el cultivo de interés, en dicho caso, el pasto.

\section{CONCLUSIONES}

En el presente estudio se encontró los efectos de 3 implementos de labranza en una zona de pastura sobre la compactación del suelo, propiedades físicas e hidropedológicas del mismo.

La labranza mecanizada del suelo mediante el palín mecánico (PM) después de
40 días resultó en un descenso de la compactación del suelo expresada como resistencia a la penetración a capacidad de campo, así como en un incremento significativo de la infiltración acumulada del mismo.

No se encontró diferencia significativa entre los 3 implementos de mecanización en la densidad aparente, la porosidad y el espacio aéreo del suelo, sin embargo estas variables difieren significativamente con respecto al tratamiento testigo. El espacio aéreo determinado para los tratamientos de mecanización incrementó en casi un $50 \%$ con respecto al tratamiento testigo. Sin embargo los valores de porosidad obtenidos para el tratamiento testigo no eran limitantes para el desarrollo radical del pasto de acuerdo con lo reportado en la literatura.

El tratamiento subsolador (S) produjo los valores más elevados de conductividad hidráulica, pero sin diferencia estadística a los restantes tratamientos. La variabilidad espacial y temporal de este parámetro se deberá tomar en cuenta para futuros estudios de mecanización, compactación e hidropedología.

\section{AGRADECIMIENTOS}

Agradecemos profundamente al personal del Ministerio de Agricultura y Ganadería (MAG) de San Mateo, en especial al Ing. Agr. Carlos Barbosa por la colaboración brindada en el desarrollo del trabajo de campo y logística. También agradecemos a la Comisión Asesora sobre Degradación de Tierras (CADETI), por el apoyo brindado durante el desarrollo del presente trabajo.

Al laboratorio de suelos del Instituto Nacional de Innovación y Transferencia en Tecnología Agropecuaria (INTA) por su colaboración en los análisis químicos del suelo.

De manera muy especial, agradecemos al Dr. Mario Morales Sánchez, profesor de la Universidad de Costa Rica sede Liberia, por las revisiones al manuscrito y sus valiosas sugerencias. 


\section{LITERATURA CITADA}

AGÜERO J.M., ALVARADO A. 1983. Compactación y compactibilidad de suelos agrícolas y ganaderos de Guanacaste, Costa Rica. Agronomía Costarricense 7(2):27-33.

ALEGRE J.C., CASSEL D.K., AMEZQUITA E. 1991. Tillage systems and soil properties in Latin America. Soil and Tillage Research 20:147-163.

ALVARADO A. 2004. Maquinaria y mecanización agrícola. EUNED. San José, Costa Rica. 612 p.

ÁLVAREZ R., STEINBACH H.S. 2009. A review of the effects of tillage systems on some soil physical properties, water content, nitrate availability and crops yield in the Argentine Pampas. Soil and Tillage Research 104(1):1-15.

BAVER L.D., GARDNER W.H., GARDNER W.R. 1972. Soil Physics. Ed. Wiley, Nueva York.

BERGOEING J.P. 1998. Geomorfología de Costa Rica. Instituto Geográfico Nacional. San José. 409 p.

BUOL S.W., SOUTHARD R.J., GRAHAM R.C., McDANIEL P.A. 2011. Soil genesis and classification. John Wiley \& Sons.

BOUMA J. 2006. Hydropedology as a powerful tool for environmental policy research. Geoderma 131(3):275-286.

BOUWER H. 1986. Intake rate: cylinder infiltrometer, pp. 825-843. In: A. Klute (ed.). Methods of Soil Analysis, Part 1. Physical and Mineralogical Properties, Monograph 9. ASA, Madison, WI.

CORPORACIÓN GANADERA. 2013. Informe encuesta ganadera 2012. San José, Costa Rica. Consultado el 11 de agosto del 2015. Disponible en http://corfoga. org/2012/wp-content/uploads/2012/09/InformePreliminar-de-Muestreo-Ganadero.pdf

DA SILVA A.P., KAY B.D., PERFECT E. 1994. Characterization of the least limiting water range of soils. Soil Science Society of America Journal 58(6):1775-1781.

DEC D., DÖRNER J., BALOCCHI O. 2011. Temporal and spatial variability of structure dependent properties of a volcanic ash soil under pasture in Southern Chile. Chilean Journal of Agricultural Research 71(2):293-303.

DEC D., DÖRNER J., BALOCCHI O., LOPEZ I. 2012. Temporal dynamics of hydraulic and mechanical properties of an Andosol under grazing. Soil \& Tillage Research 125:44-51.

DEXTER A.R., CZYŻ E.A., GAŢE O.P. 2004. Soil structure and the saturated hydraulic conductivity of subsoils. Soil and Tillage Research 79(2):185-189.
DEXTER A.R., RICHARD G. 2009. The saturated hydraulic conductivity of soils with n-modal pore size distributions. Geoderma 154(1):76-85.

DREWRY J.J., PATON R.J. 2000. Effect of subsoiling on soil physical properties and dry matter production on a Brown Soil in Southland, New Zealand, New Zealand Journal of Agricultural Research 43(2):259268.

FORSYTHE W. 1985. Física de suelos. Manual de laboratorio. IICA, Costa Rica. 212 p.

FORSYTHE W. 1997. Las condiciones físicas, la producción agrícola y la calidad del suelo. Agronomía Costarricense 21:35-47.

FORSYTHE W., TAFUR N. 1985. Efecto de varios métodos para la preparación del terreno sobre la resistencia a la penetración y sobre el rendimiento del maíz (Zea mays L.), yuca (Manihot esculenta Crantz) y camote (Ipomoea batatas L.) en asociación. I. Efecto en el suelo del sistema de cosecha y de preparación de la tierra. Turrialba 35(4):357-370.

FORSYTHE W., SANCHO F., VILLATORO M. 2005. Efecto de la compactación de suelos sobre el rendimiento del maíz en tres localidades de Costa Rica. Agronomía Costarricense 29(3):175-185.

FORSYTHE W., SCHWEIZER S. 2001. La resistencia a la penetración y la rata de infiltración como indicadores de las condiciones físicas de un suelo de Costa Rica. In: XV Congreso Latinoamericano y V Cubano de la Ciencia del Suelo. Varadero, 11 al 16 de noviembre, 2001. CD-ROM.

GÓMEZ J.A., GIRÁLDEZ J.V., PASTOR M., FERERES E. 1999. Effects of tillage method on soil physical properties, infiltration and yield in an olive orchard. Soil and tillage research 52(3):167-175.

GUENNI O., MARÍN D., BARUCH Z. 2002. Responses to drought of five Brachiaria species. I. Biomass production, leaf growth, root distribution, water use and forage quality. Plant and soil 243(2):229-241.

GUZHA A.C. 2004. Effects of tillage on soil microrelief, surface depression storage and soil water storage. Soil and Tillage Research 76(2):105-114.

HARGREAVES G.H., SAMANI Z.A. 1985. Reference crop evapotranspiration from temperature. Applied Eng. in Agric 1(2):96-99.

HOLDRIDGE L.R. 1967. Life zone ecology. Tropical Science Center. San José, Costa Rica. 206 p.

HORNE D.J., ROSS C.W., HUGHES K.A. 1992. Ten years of a maize/oats rotation under three tillage systems on a silt loam in New Zealand. 1. A comparison of some soil properties. Soil and Tillage Research 22(1):131-143. 
INGELMO F., MOLINA M., DE PAZ J.M., VISCONTI F. 2011. Soil saturated hydraulic conductivity assessment from expert evaluation of field characteristics using an ordered logistic regression model. Soil and Tillage Research 115:27-38.

KAY B.D., VANDENBYGAART A.J. 2002. Conservation tillage and depth stratification of porosity and soil organic matter. Soil and Tillage Research 66(2):107118.

KAUFFMAN J.B., KRUEGER W.C. 1984. Livestock impacts on riparian ecosystems and streamside management implications. A review. Journal of Range Management 37(5):430-438.

KOSTIAKOV A.N. 1932. On the dynamics of the coefficient of water-percolation in soils and on the necessity for studying it from a dynamic point of view for purposes of amelioration. Transactions 6th commission pp. 17-21.

LETEY J. 1985. Relationship between soil physical properties and crop production. Advances in Soil Science 1:277-294.

LICHT M.A., AL-KAISI M. 2005. Strip-tillage effect on seedbed soil temperature and other soil physical properties. Soil and Tillage research 80(1):233-249.

LIN H. 2003. Hydropedology. Vadose Zone Journal 2(1):1-11.

LIN H. 2012. Hydropedology: Addressing fundamentals and building bridges to understand complex pedologic and hydrologic interactions. Hydropedology: Synergistic Integration of Soil Science and Hydrology pp. 3-39.

LIN H., BOUMA J., PACHEPSKY Y., WESTERN A., THOMPSON J., VAN GENUCHTEN R., HANSJORG V., LILLY A. 2006. Hydropedology: Synergistic integration of pedology and hydrology. Water Resources Research 42(5):1-13.

LIPIEC J., KUŚ J., SŁOWIŃSKA A., NOSALEWICZ A. 2006. Soil porosity and water infiltration as influenced by tillage methods. Soil and Tillage research 89(2):210-220.

LISTO E. 2009. Balance hídrico en cuencas forestales prioritarias para el abastecimiento de agua en Centroamérica. Proyecto Fin de Carrera de la ETSI Montes. Universidad Politécnica de Madrid. Madrid, España. 155 p.

MARTÍNEZ L.J., ZINCK J.A. 2004. Temporal variation of soil compaction and deterioration of soil quality in pasture areas of Colombian Amazonia. Soil and Tillage Research 75(1):3-18.

MINISTERIO DE AGRICULTURA Y GANADERIA. 2000. Análisis del censo ganadero 2000. San José, Costa Rica. Consultado el 6 de febrero del 2015. Disponible en http://www.mag.go.cr/biblioteca virtual_animal/censo-ganadero-2000.pdf

MUBARAK I., ANGULO R., MAILHOL J.C., RUELLE P., KHALEDIAN M., VAUCLIN M. 2010. Spatial analysis of soil surface hydraulic properties: Is infiltration method dependent? Agricultural water management 97(10):1517-1526.

MWENDERA E.J., SALEEM M.A. 1997. Infiltration rates, surface runoff, and soil loss as influenced by grazing pressure in the Ethiopian highlands. Soil use and management 13(1):29-35.

NAKANO K., MIYAZAKI T. 2005. Predicting the saturated hydraulic conductivity of compacted subsoils using the non-similar media concept. Soil and Tillage Research 84(2):145-153.

PACHEPSKY Y.A., RAWLS W.J., LIN H.S. 2006. Hydropedology and pedotransfer functions. Geoderma 131(3):308-316.

PARIPOVIC D. 2011. Impacts of conversion from forestry to pasture on soil physical properties of Vitrands (Pumice Soils) in the Central North Island, New Zealand. M.Sc. Thesis. The University of Waikato, New Zealand. $91 \mathrm{p}$.

PIERCE F.J., FORTIN M.C., STATON M.J. 1992. Immediate and residual effects of zone-tillage in rotation with no-tillage on soil physical properties and corn performance. Soil Tillage Res 24:149-165.

PIETOLA L., HORN R., YLI-HALLA M. 2005. Effects of trampling by cattle on the hydraulic and mechanical properties of soil. Soil and tillage research 82(1):99108

PRITCHETT K., KENNEDY A.C., COGGER C.G. 2011. Management effects on soil quality in organic vegetable systems in western Washington. Soil Science Society of America Journal 75(2):605-615.

RIENZNER M., GANDOLFI C. 2014. Investigation of spatial and temporal variability of saturated soil hydraulic conductivity at the field-scale. Soil and Tillage Research 135:28-40.

SARRANTONIO M., MOLLOY T. 2003. Response of sweet corn to red clover under two tillage methods. Journal of Sustainable Agriculture 23(2):91-109.

ARROW S.H. 2007. Soil compaction by grazing livestock in silvopastures as evidenced by changes in soil physical properties. Agroforest Syst 71:215-223.

SKINNER A.K., LUNT I.D., SPOONER P., McINTYRE S. 2009. The effect of soil compaction on germination and early growth of Eucalyptus albens and an exotic annual grass. Austral Ecology 34(6):698-704.

SOIL SURVEY STAFF. 2014. Keys to Soil. Twelfth edition. NRCS-USDA. USA. 360 p.

SOJKA R.E., HORNE D.J., ROSS C.W., BAKER C.J. 1997. Subsoiling and surface tillage effects on soil physical properties and forage oat stand and yield. Soil and Tillage Research 40(3):125-144.

SOLANO J., VILLALOBOS R. 2012. Regiones y Subregiones Climáticas De Costa Rica. San José, Costa Rica. Consultado el 11 de agosto del 2015. Disponible en http://www.imn.ac.cr/publicaciones/ estudios/Reg_climaCR.pdf 
STRUDLEY W., GREEN T.R., ASCOUGH II J.C. 2008. Tillage effects on soil hydraulic properties in space and time: State of the science. Soil and Tillage Research 99(1):4-48.

TRIMBLE S.W., MENDEL A.C. 1995. The cow as a geomorphic agent - a critical review. Geomorphology 13(1):233-253.

WARREN S.D., THUROW T.L., BLACKBURN W.H., GARZA N.E. 1986. The influence of livestock trampling under intensive rotation grazing on soil hydrologic characteristics. Journal of Range Management 39(6):491-495.

WHEELER M.A., TRLICA M.J., FRASIER G.W., REEDER J.D. 2002. Seasonal Grazing Affects Soil Physical Properties of a Montane Riparian Community. Journal of Range Management 55(1):49-56.

XU D., MERMOUD A. 2003. Modeling the soil water balance based on time-dependent hydraulic conductivity under different tillage practices. Agric. Water Manage 63(2):139-151. 
